\title{
Acute heart failure in pregnancy: importance of a timely multidisciplinary approach to recognition and management
}

\author{
SANOJ CHACKO ${ }^{1,2}$, JOSEPH DE BONO ${ }^{2}$, HOWARD MARSHALL ${ }^{2}$, YUMNA B. HASEEB ${ }^{1}$, SOHAIB HASEEB ${ }^{1}$, \\ KATIE MORRIS ${ }^{2}$, SARA THORNE ${ }^{2}$

\footnotetext{
${ }^{1}$ Heart Rhythm Service, Kingston Health Sciences Centre, Queen's University, Kingston, Ontario, Canada
} \\ ${ }^{2}$ Queen Elizabeth Hospital, Mindelsohn Way, Birmingham, United Kingdom
}

\begin{abstract}
Increased cardiovascular demands of pregnancy may unmask occult diseases, such as dilated cardiomyopathy or valvular stenosis, or precipitate peripartum cardiomyopathy. We report a case of the emergency management and delivery of a young pregnant woman who presented with acute decompensated heart failure that was not immediately recognized. An emergency transfer to a tertiary care institution was arranged. Once diagnosed, the patient received multidisciplinary care shared between cardiologists, obstetricians, cardiac anesthetists, a neonatologist, and a midwife, resulting in good maternal and fetal outcomes.
\end{abstract}

Key words: acute disease, heart failure, female, emergency care, pregnancy.

What is new? What is important?

Young women may appear superficially well compensated in the face of severe hemodynamic compromise. Rapid early treatment and referral to a center with a highly functional multidisciplinary cardiac obstetric team is paramount to avoid catastrophic circulatory collapse.

\section{INTRODUCTION}

Cardiac disease is a common cause of indirect maternal mortality [1]. Increased cardiovascular demands of pregnancy may unmask occult diseases, such as dilated cardiomyopathy or valvular stenosis, or precipitate peripartum cardiomyopathy [2]. Therefore, obstetric and acute medical teams should be able to recognize the symptoms and signs of decompensating heart disease.

Pregnancy is associated with significant physiological changes and cardiovascular stress, with a $50 \%$ increase in blood volume and cardiac output before the end of the second trimester in a singleton pregnancy. The rise in cardiac output is achieved by a $30 \%$ increase in stroke volume and a $10 \%$ rise in heart rate. The increased blood volume is accommodated by vasodilation, which results in a fall in systemic and pulmonary vascular resistance, and by low resistance uteroplacental circulation [3]. Women whose cardiovascular impairments limit their ability to increase cardiac output, such as those with systolic ventricular dysfunction or obstructive valve lesions, are at high risk of cardiac decompensation by the end of the second trimester [4].

We report a case of the emergency management and delivery of a young pregnant woman who presented with acute decompensated heart failure (HF).

\section{Case}

A 21-year-old previously well primigravida, 28 weeks pregnant with twins, presented to her local hospital with a three-day history of increasing breathlessness, orthopnea, and paroxysmal nocturnal dyspnea. On arrival to the emergency department, she was unable to talk in full sentences, was warm and well perfused with sinus tachycardia of $130 \mathrm{bpm}$, and had a blood pressure 130/80 mmHg. No murmur was recorded. She had fine inspiratory crackles and wheeze and reduced air entry. A diagnosis of pulmonary embolism was considered, and 
computed tomography (CT) pulmonary angiogram was arranged. However, she could not lie flat and was only able to tolerate a scout film, which showed pulmonary congestion. She was admitted and treated with oxygen, $1.5 \mathrm{mg} / \mathrm{kg}$ of enoxaparin and antibiotics, as well as betamethasone for fetal lung maturation. She slept upright and was noted to be more breathless the following morning. An echocardiogram showed a dilated left ventricle with impaired systolic function and mitral regurgitation.

The local obstetrician arranged an emergency transfer to our institution. On arrival, she was tachycardic, orthopneic, and unable to speak in full sentences. As part of the preliminary assessment for heart failure, a chest radiography was performed which confirmed acute pulmonary edema (Figure 1), and blood tests revealed an elevated B-type natriuretic peptide (BNP) of 2100 $\mathrm{pg} / \mathrm{mL}$. Electrocardiogram showed sinus tachycardia. An echocardiogram confirmed global severe left ventricular systolic dysfunction with an ejection fraction of $<25 \%$ and severe functional mitral regurgitation (Figure 2). Ultrasound showed that both fetuses were alive with normal heartbeats. She was treated with intravenous diuretics, a glyceryl-trinitrate infusion, and continuous positive airway pressure ventilation. She was given further intravenous furosemide and a glyceryl trinitrate infusion.

A multidisciplinary team led by a cardiologist and maternal medicine obstetrician, and including obstetric and cardiac anesthetists, a neonatologist, and a midwife, were assembled. The patient and the twin fetuses were closely monitored in the coronary care unit, equipped with emergency delivery and neonatal resuscitation facilities.

The patient made rapid improvement after the initial bolus dose of intravenous diuretics and continuous glyceryl-trinitrate infusion.
Within 3 hours of arrival, she looked well and was able to speak freely and lie flat. However, she remained tachycardic at $130 \mathrm{bpm}$ and repeat blood tests at 2 hours and 4 hours post admission showed a gradual decline when compared to her baseline investigations on arrival (Table 1). She had evidence of increasing metabolic acidosis indicating inadequate tissue and renal perfusion. These concerns prompted a decision to deliver the woman in cardiac theatres forthwith. A cardiac ICU bed was arranged, as well as two neonatal teams and transport incubators. A cardiac surgeon and theatre team were on standby in case she required mechanical support postdelivery. An intra-aortic balloon pump was implanted to provide hemodynamic support during delivery.

She underwent an uncomplicated emergency caesarean under general anesthesia and delivered twins. Notably, the twins were premature (28 weeks) but otherwise healthy. Post-delivery transesophageal echocardiography showed no improvements in ventricular function, but a reduction in left ventricular dimensions and in the severity of her functional mitral regurgitation to mild. She required no pharmacological ionotropic support, and the balloon pump was explanted at 24 hours postdelivery. Post-delivery cardiac MRI confirmed poor dilated cardiomyopathy but did not reveal an underlying cause. There was no left ventricular thrombus and no regional wall abnormalities. She was discharged one-week post-delivery on an angiotensin converting enzyme inhibitor and low dose beta blocker.

At follow-up six weeks later, there was no recovery in ventricular function. She was given contraceptive advice and counseled that a further pregnancy would carry a high risk of maternal mortality and fetal morbidity.

Table 1

Deterioration in renal and metabolic status despite apparent clinical improvement

\begin{tabular}{|c|c|c|c|c|}
\hline & Pre-transfer & On arrival & 2h post-transfer & 4h post-transfer \\
\hline $\begin{array}{c}\text { Potassium } \\
(\mathbf{m m o l} / \mathbf{L})\end{array}$ & 4.7 & 5.0 & 5.3 & 5.9 \\
\hline $\begin{array}{c}\text { Creatinine } \\
(\mathbf{u m o l} / \mathbf{L})\end{array}$ & 48 & 59 & 65 & 71 \\
\hline $\begin{array}{c}\text { Base excess } \\
(\mathbf{m E q} / \mathbf{L})\end{array}$ & -3.6 & -7.6 & -7.8 & -8.5 \\
\hline
\end{tabular}




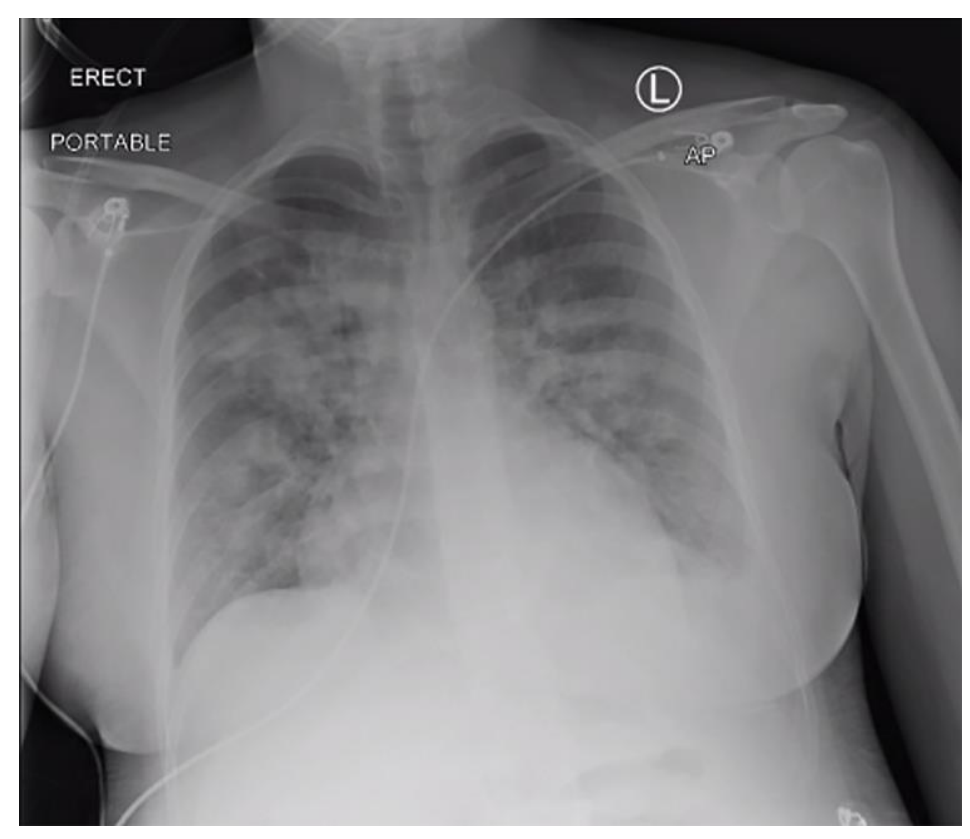

Figure 1.

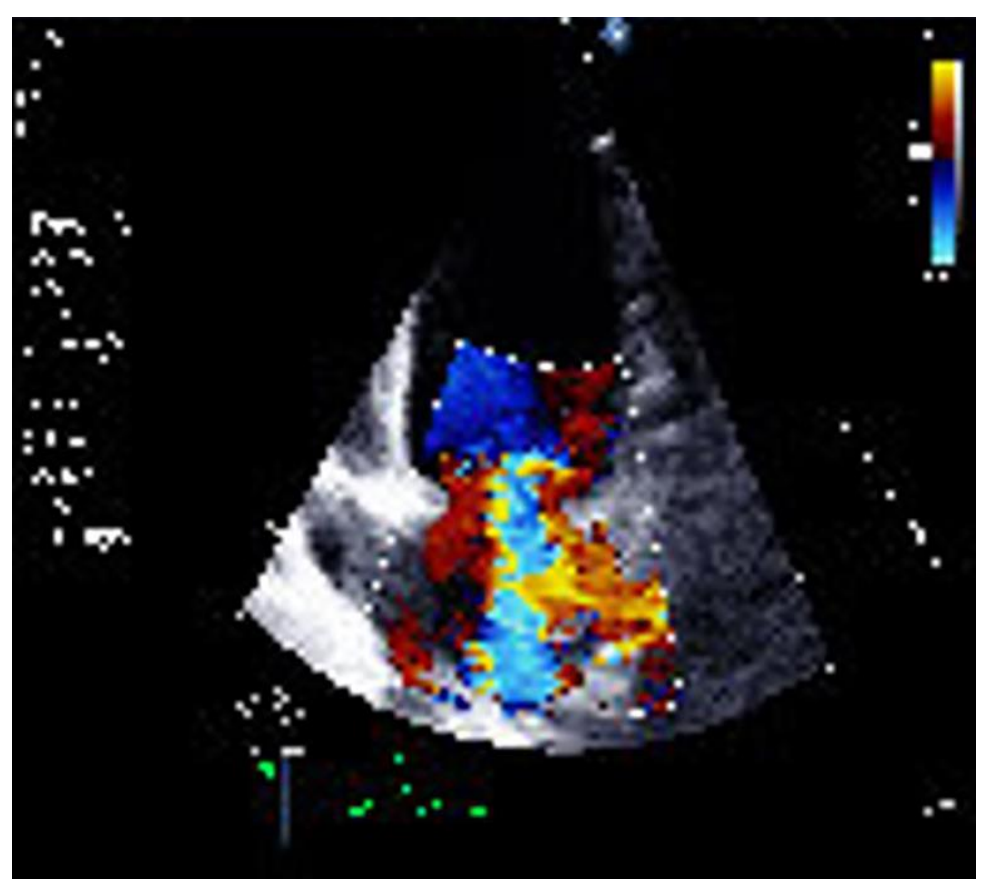

Figure 2.

\section{DISCUSSION}

This patient's initial presentation emphasizes the importance of recognizing the signs and symptoms of decompensating heart disease in pregnancy. Although breathlessness and tachycardia may be normal in pregnancy, orthopnea and sinus tachycardia $>100$ are not, and require a positive diagnosis [5]. Orthopnea, in particular, demands a search for cardiac disease [6]. Our patient's inability to lie flat in the CT scanner should have flagged pulmonary edema as the cause of breathlessness.

Young, previously well pregnant women with undiagnosed heart disease may compensate for a considerable time and have relatively 
minor symptoms of breathlessness that are easily mistaken for 'normal pregnancy.' When they finally decompensate with overt HF, it is incumbent on the multidisciplinary team to make an accurate diagnosis and act appropriately. Our patient presented at her local hospital at the start of a bank holiday weekend. She had good response to the initial treatment of pulmonary edema, and consideration could have been given to delaying her delivery until after the weekend when gathering an extensive multidisciplinary team would be easier. However, her continuing tachycardia and biochemical evidence of inadequate tissue and renal perfusion demonstrated that she remained in a decompensated state, and delivery could not be delayed.

A contemporary approach to the management of HF presenting during pregnancy has been previously outlined [5]. In women with a moderate or high risk of complications during pregnancy, the European Society of Cardiology recommends pre-pregnancy counseling and multidisciplinary management by an expert center [7]. HF presenting during pregnancy is associated with unfavorable fetal outcomes, including premature and low birth weight, and an increased risk of maternal death [8]. While the management of our case adheres to the standard guidelines, the case also highlights the importance of a timely well-coordinated multidisciplinary approach that may influence a favorable outcome.

The underlying cause of her dilated cardiomyopathy remains uncertain. Peripartum cardiomyopathy is defined as occurring in the last month of a term pregnancy or the first six months post-partum, too early to be considered in this case [6]. The alternative diagnosis would be a preexisting dilated cardiomyopathy unmasked by the demands of her twin pregnancy.

\section{CONCLUSION}

Our patient presented with acute decompensated HF that was not immediately recognized. Once diagnosed, she was transferred rapidly to a centre with appropriate expertise and received high-quality multidisciplinary care, resulting in good maternal and fetal outcomes.

Correspondence to: Sanoj Chacko, MD, Attending Cardiac Electrophysiologist, Cardiac Electrophysiology and Pacing, 76 Stuart St, Kingston Health Sciences Centre K7L 2V7, Queen's University, Kingston, Ontario, Canada

Acknowledgements: None. Email: Sanoj.Chacko@kingstonhsc.ca

Consent: Informed consent was given by the patient prior to the publication of this case report.

Conflict of interest disclosure: The authors declare that there are not conflicts of interest.

\section{REFERENCES}

1. LAMEIJER H., SCHUTTE JM., SCHUITEMAKER NWE., van Roosmalen JJM., Pieper PG. Maternal mortality due to cardiovascular disease in the Netherlands: a 21-year experience. Neth Heart J. 2020;28 (1):27-36.

2. AHMAD WAW., KHANOM M., YAAKOB ZH. Heart failure in pregnancy: an overview. Int J Clin Pract. 2011;65(8):848-51.

3. SANGHAVI M., RUTHERFORD JD. Cardiovascular Physiology of Pregnancy. Circulation. 2014;130(12):1003-8.

4. SAFI LM., TSIARAS SV. Update on Valvular Heart Disease in Pregnancy. Curr Treat Options Cardiovasc Med. $2017 ; 19(9): 70$.

5. ANTHONY J., SLIWA K. Decompensated Heart Failure in Pregnancy. Card Fail Rev. 2016;2(1):20-6.

6. SLIWA K., HILFIKER-KLEINER D., PETRIE MC., MEBAZAA A., PIESKE B., BUCHMANN E., et al. Current state of knowledge on aetiology, diagnosis, management, and therapy of peripartum cardiomyopathy: a position statement from the Heart Failure Association of the European Society of Cardiology Working Group on peripartum cardiomyopathy. Eur J Heart Fail. 2010;12(8):767-78.

7. REGITZ-ZAGROSEK V., ROOS-HESSELINK JW., BAUERSACHS J., BLOMSTRÖM-LUNDQVIST C., CÍFKOVÁ R., DE BONISM., et al. 2018 ESC Guidelines for the management of cardiovascular diseases during pregnancy. Eur Heart J. 2018;39(34):3165-241.

8. NG AT., DUAN L., WIN T., SPENCER HT., LEE MS. Maternal and fetal outcomes in pregnant women with heart failure. Heart. 2018;104(23):1949-54.

Received $13^{\text {th }}$ February 2021 